\title{
Synthesis, anti-HIV activity and molecular modeling study of some new pyrimidine analogues
}

\author{
Yossra Abood Marich, Niran Jassim Al-Salihi and Najim Aboud Al-Masoudi * \\ Department of Chemistry, College of Science, University of Basrah, Basrah, 61001, Iraq \\ *Corresponding author at: Department of Chemistry, College of Science, University of Basrah, Basrah, 61001, Iraq. \\ Tel.: +49.75.3134435. Fax: +49.75.3134435. E-mail address: najim.al-masoudi@gmx.de (N.A. Al-Masoudi).
}

\section{ARTICLE INFORMATION}

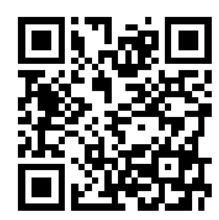

DOI: $10.5155 /$ eurjchem.5.4.588-594.1109

Received: 15 June 2014

Received in revised form: 08 July 2014

Accepted: 08 July 2014

Online: 31 December 2014

\section{KEYWORDS}

Pyrimidines

Anti-HIV activity

Sodium hypochlorite

Molecular modeling study

Structure activity relationship

Non-nucleoside reverse transcriptase inhibitors

\section{Introduction}

Pyrimidine and its derivatives demonstrated a diverse array of biological and pharmacological activities including antitumor [1-6], antimicrobial [7-10], and antihypertensive [11] in addition to their cardiovascular $[12,13]$ and diuretic $[14,15]$ properties. Some pyrimidine analogues exhibited potent antiviral activity against a wide spectrum of unrelated viruses, such as poliovirus [16] and herpes virus [17] and antiHIV agents [18-20], whereas two recent diarylpyrimidines (DAPY), rilpivirine $\mathbf{1}$ [21] and etravirine $\mathbf{2}$ [22, 23] have been classified as non-nucleoside reverse transcriptase inhibitors (NNRTI's), meanwhile Chen et al. [24] have reported a new class of diarylpyrimidines (CHX-DAPYs) as potent NNRTI's. Further, several pyrimidine derivatives exhibited significant antitumor activity e.g. imatinib mesylate (Gleevec, 3) [25], an interesting novel agent for the treatment of chronic Leukemia is the tyrosine kinase inhibitor which contains a 4-pyridylsubstituted pyrimidine-2-amine, in addition to a 2,4-diamino$N^{4}$-6-diaryl-pyrimidines where the latter were identified to block the proliferation of tumor cell lines in vivo, especially duodenum cancer [26]. Monastrol 4 [27] is another model of pyrimidine derivative as inhibitor of kinesin Eg5 that interact with microtubuline and then causes mitotic arrest [28] (Figure $1)$.
Recently, Kim et al. [29] have reported some novel pyrimidine derivatives as potent acid pump antagonists (APAs). Jian et al. [30] have reviewed the biological and medicinal significance of pyrimidines extensively.<smiles>Cc1cc(/C=C/C#N)cc(C)c1Nc1ccnc(Nc2ccccc2)n1</smiles>

1. Rilpivirine

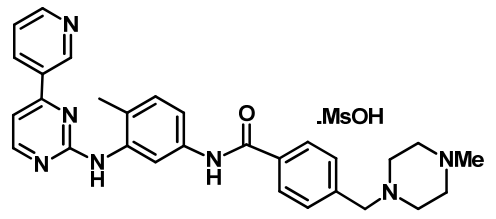

3. Gleevec<smiles>Cc1cc(C#N)cc(C)c1Nc1nc(Nc2ccc(C#N)cc2)nc(N)c1Br</smiles>

2. Etravirine<smiles>CCOC(=O)C1=C(C)NC(=S)NC1(C(=O)OCC)c1cccc(O)c1</smiles>

4. Monastrol

Figure 1. Pyrimidine analogues antitumor and anti-HIV drugs. 


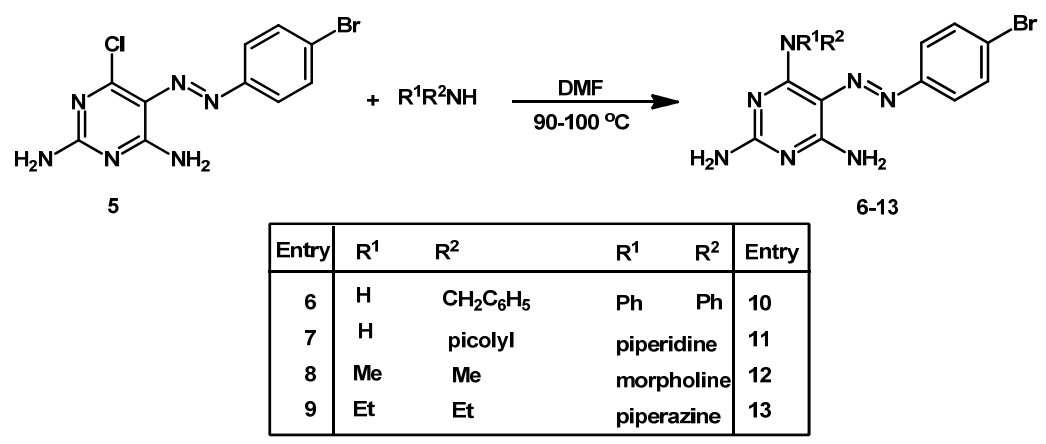

Scheme 1

In continuation of our ongoing work on the synthesis of new anti-HIV pyrimidine derivatives [31,32], we report here the synthesis of new of series of pyrimidines having substituted amino and thio groups with evaluation of their anti-HIV activity as well the SAR and molecular modeling study.

\section{Experimental}

\subsection{Instrumentation}

Melting points are uncorrected and were measured on a Büchi melting point apparatus B-545 (Büchi Labortechnik AG, Switzerland). NMR data were obtained on 400 and $600 \mathrm{MHz}$ $\left({ }^{1} \mathrm{H}\right)$ and $150.91 \mathrm{MHz}\left({ }^{13} \mathrm{C}\right)$ spectrometers (Avance III, Bruker, Germany) with TMS as internal standard and on the $\delta$ scale in ppm. Heteronuclear assignments were verified by ${ }^{1} \mathrm{H},{ }^{13} \mathrm{C}$ HMBC and ${ }^{1} \mathrm{H},{ }^{13} \mathrm{C}$ HSQC NMR experiments. Microanalytical data were obtained with a Vario, Elemental analyzer (Shimadzu, Japan). Analytical silica gel TLC plates $60 \mathrm{~F}_{254}$ were purchased from Merck. Microwave supported reaction was performed in a SmithSynthesizer (temperature control of irradiation power up to $800 \mathrm{~W}$ ). All reagents were obtained from commercial suppliers and were used without further purification.

\subsection{Synthesis}

\subsubsection{General procedure for the preparation of 2,6-diamino- 4-alkylamino-5-((p-bromophenyl)diazenyl)pyrimidine derivatives (6-13)}

A solution of compound 5 (164 mg, $0.50 \mathrm{mmol}$ ) in DMF (20 $\mathrm{mL})$ and an appropriate amine $(1.00 \mathrm{mmol})$ was heated in an oil bath at $90-100{ }^{\circ} \mathrm{C}$ for $4-5 \mathrm{~h}$. Then water $(25 \mathrm{~mL})$ was added, the solution was cooled, and the yellow precipitate was collected, washed with water, and dried. Recrystallization from ethanol afforded the desired product (Scheme 1).

2,6-Diamino-4-benzylamino-5-p-bromophenylazopyrimidine (6): From benzylamine (107 mg). Yield: $110 \mathrm{mg}$ (55\%). M.p.: $160-167{ }^{\circ} \mathrm{C} . R_{\mathrm{f}}=0.75 .{ }^{1} \mathrm{H}$ NMR $\left(400 \mathrm{MHz}, \mathrm{DMSO}-d_{6}, \delta, \mathrm{ppm}\right)$ : 8.84-8.14 (br. s., $2 \mathrm{H}, \mathrm{NH}_{2}$ ), 7.72 (d, $2 \mathrm{H}, J=7.9 \mathrm{~Hz}, \mathrm{H}-\mathrm{Ar}$ ), 7.54 (d, $2 \mathrm{H}, J=7.9 \mathrm{~Hz}, \mathrm{H}-\mathrm{Ar}), 7.36-7.24\left(\mathrm{~m}, 6 \mathrm{H}, \mathrm{H}-\mathrm{Ar}+\mathrm{CH}_{2} \mathrm{NH}\right), 6.62(\mathrm{br}$ s., $2 \mathrm{H}, \mathrm{NH}_{2}$ ), 4.72 (d, $\left.2 \mathrm{H}, J=6.5 \mathrm{~Hz}, \mathrm{CH}_{2}\right) .{ }^{13} \mathrm{C}$ NMR $(150.91 \mathrm{MHz}$,

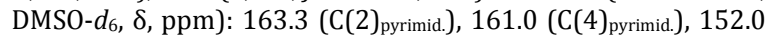
(C(6) pyrimid.), 139.0, 131.7, 128.3, 127.3, 126.9 (C Carom.) $_{12} 12.7$ (C$\mathrm{Br}), 119.1$ ( $\mathrm{C}_{\text {arom. }}$ ), 110.1 (C(5) $\left.)_{\text {pyrimid. }}\right) ; 2.9\left(\mathrm{CH}_{2}\right)$. Anal. calcd. for $\mathrm{C}_{17} \mathrm{H}_{16} \mathrm{BrN}_{7}$ : C, 51.27; H, 4.05; N, 24.62. Found: C, 51.04; H, 3.92; N $24.41 \%$.

2,6-Diamino-5-((p-bromophenyl)diazenyl)-4-(2-picolyl amino)pyrimidine (7): From 2-picolylamine (108 mg). Yield: 64 mg (32\%). M.p.: 193-196 ${ }^{\circ} \mathrm{C} . R_{\mathrm{f}}=0.54 .{ }^{1} \mathrm{H}$ NMR $(400 \mathrm{MHz}$, DMSO- $\left.d_{6}, \delta, \mathrm{ppm}\right): 8.50\left(\mathrm{~d}, 2 \mathrm{H}, J=4.8 \mathrm{~Hz}, \mathrm{NH}_{2}\right), 8.36(\mathrm{~d}, 1 \mathrm{H}, J=$ $8.2 \mathrm{~Hz}, \mathrm{H}$ picolyl-6")-Ar), 7.78 (m, 3H, Harom.-3' + Harom.-5' + Hpicolyl4"), 7.55 (d, 2H, J = $\left.8.2 \mathrm{~Hz}, \mathrm{H}_{\text {picolyl }}-3 "+\mathrm{H}_{\text {picolyl }}-5 "\right), 7.31$ (d, 2H, $J=$
$8.0 \mathrm{~Hz}, \mathrm{H}_{\text {arom. }}-2^{\prime}+\mathrm{H}_{\text {arom. }}-6^{\prime}$ ), 6.60 (br s., $2 \mathrm{H}, \mathrm{NH}_{2}$ ), 4.73 (d, $2 \mathrm{H}$, $\left.J_{\mathrm{NH}, \mathrm{CH} 2}=6.1 \mathrm{~Hz}, \mathrm{HNCH}_{2}\right), 3.28\left(\mathrm{~d}, 1 \mathrm{H}, \mathrm{HNCH}_{2}\right) .{ }^{13} \mathrm{C} \mathrm{NMR}(150.91$,

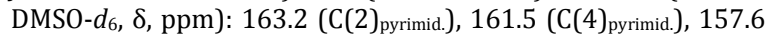
(C(2') picolyl $), \quad 152.0 \quad$ (C(6) (C(4')picolyl), 131.7, 122.9, 122.1 (Carom.), 119.3 (C(3') picolyl +

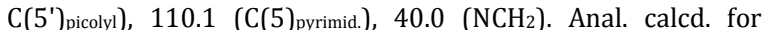
$\mathrm{C}_{16} \mathrm{H}_{15} \mathrm{BrN}_{8}: \mathrm{C}, 48.13$; H, 3.79; N, 28.07. Found: C, 47.91; H, 3.68; $\mathrm{N}, 27.81 \%$.

2,6-Diamino-5-((p-bromophenyl)diazenyl)-4-dimethylamino pyrimidine (8): From dimethylamine $(45 \mathrm{mg})$. Yield: $124 \mathrm{mg}$ (74\%). M.p.: $204-207^{\circ} \mathrm{C}$ (Dec.). $R_{\mathrm{f}}=0.55 .{ }^{1} \mathrm{H}$ NMR $(400 \mathrm{MHz}$, DMSO- $d_{6}, \delta$, ppm): 7.79 (br s., $2 \mathrm{H}, \mathrm{NH}_{2}$ ), $7.61(\mathrm{~d}, 2 \mathrm{H}, J=7.9 \mathrm{~Hz}$, $\mathrm{H}-\mathrm{Ar}$ ), 7.54 (d, $2 \mathrm{H}, J=7.9 \mathrm{~Hz}, \mathrm{H}-\mathrm{Ar}$ ), 6.81 (br s., $2 \mathrm{H}, \mathrm{NH}_{2}$ ), 3.31 (s, 6H, $\mathrm{NMe}_{2}$ ). ${ }^{13} \mathrm{C}$ NMR (DMSO- $d_{6}, \delta, \mathrm{ppm}$ ): 163.0 (C(2) pyrimid.),

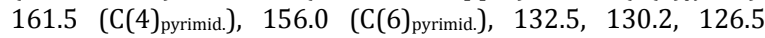
(Carom.), 122.9 (C-Br), 104.1 (C(5) $)_{\text {pyrimid.), }} 39.1\left(\mathrm{NMe}_{2}\right.$ ). Anal. calcd. for $\mathrm{C}_{12} \mathrm{H}_{14} \mathrm{BrN}_{7}$ : C, 42.87; $\mathrm{H}, 4.20 ; \mathrm{N}, 29.16$. Found: C, 42.66; H, 4.08; N, $28.98 \%$.

2,6-Diamino-5-((p-bromophenyl)diazenyl)-4-diethylamino pyrimidine (9): From diethylamine $(73 \mathrm{mg})$. Yield: $126 \mathrm{mg}$ (69\%). M.p.: $145-147^{\circ} \mathrm{C}(\mathrm{Dec}) . R_{\mathrm{f}}=0.66 .{ }^{1} \mathrm{H}$ NMR $(400 \mathrm{MHz}$, DMSO- $d_{6}, \delta, p p m$ ): 9.14 (br s., $2 \mathrm{H}, \mathrm{NH}_{2}$ ), 7.72 (d, $2 \mathrm{H}, J=7.9 \mathrm{~Hz}$, $\mathrm{H}-\mathrm{Ar}$ ), 7.68 (d, $2 \mathrm{H}, J=7.9 \mathrm{~Hz}, \mathrm{H}-\mathrm{Ar}$ ), 6.60 (br s., $2 \mathrm{H}, \mathrm{NH}_{2}$ ), 3.99 (q, $\left.4 \mathrm{H}, J=7.1 \mathrm{~Hz}, 2 \mathrm{xCH}_{2} \mathrm{CH}_{3}\right), 1.23\left(\mathrm{t}, 6 \mathrm{H}, 2 \mathrm{xCH}_{2} \mathrm{CH}_{3}\right) \cdot{ }^{13} \mathrm{C} \mathrm{NMR}$ $\left(150.91, \quad\right.$ DMSO- $\left.d_{6}, \quad \delta, \quad \mathrm{ppm}\right): 163.0 \quad\left(\mathrm{C}(2)_{\text {pyrimid. }}\right), \quad 162.0$ (C(4) pyrimid.), 157.2 (C(6) pyrimid.), 132.1, 130.6, 127.9 (Carom.), $121.6 \quad(\mathrm{C}-\mathrm{Br}), \quad 100.0 \quad$ (C(5) $)_{\text {pyrimid. }),} 41.6 \quad\left(2 \mathrm{xCH}_{2} \mathrm{CH}_{3}\right), \quad 11.4$ $\left(2 \mathrm{xCH}_{2} \mathrm{CH}_{3}\right)$. Anal. calcd. for $\mathrm{C}_{14} \mathrm{H}_{18} \mathrm{BrN}_{7}$ : C, 46.16; $\mathrm{H}, 4.98 ; \mathrm{N}$, 29.92. Found: C, $45.89 ; \mathrm{H}, 4.88 ; \mathrm{N}, 28.68 \%$.

2,6-Diamino-5-((p-bromophenyl)diazenyl)-4-diphenylamino pyrimidine (10): From diphenylamine $(169 \mathrm{mg})$. Yield: $83 \mathrm{mg}$ (36\%). M.p.: $155-158{ }^{\circ} \mathrm{C}$ (Dec). $R_{\mathrm{f}}=0.58 .{ }^{1} \mathrm{H}$ NMR $(400 \mathrm{MHz}$, DMSO- $d_{6}, \delta, \mathrm{ppm}$ ): 8.19 (br s., $2 \mathrm{H}, \mathrm{NH}_{2}$ ), 7.64-7.08 (m, $14 \mathrm{H}, \mathrm{H}-$ Ar), 6.81 (br s., $2 \mathrm{H}, \mathrm{NH}_{2}$ ). ${ }^{13} \mathrm{C}$ NMR (150.91, DMSO- $d_{6}, \delta, \mathrm{ppm}$ ): 163.1 (C(2) pyrimid.), 161.3 (C(4) pyrimid.), 156.0 (C(6) pyrimid.), 140.8, 138.8, 132.6, 130.2, 129.6, 129.2, 127.3 (Carom.), 123.2 (C-Br), 104.8 (C(5) pyrimid.). Anal. calcd. for $\mathrm{C}_{22} \mathrm{H}_{18} \mathrm{BrN}_{7}$ : C, 57.40; $\mathrm{H}, 3.94$; N, 21.30. Found: C, 57.18; H, 3.82; N, $21.06 \%$.

2,6-Diamino-4-piperidino-5-p-bromophenylazo-pyrimidine (11): From piperidine (85 mg). Yield: $167 \mathrm{mg}$ (89\%). M.p.: 190$197^{\circ} \mathrm{C} . R_{\mathrm{f}}=0.90 .{ }^{1} \mathrm{H}$ NMR $\left(400 \mathrm{MHz}, \mathrm{DMSO}-d_{6}, \delta, \mathrm{ppm}\right): 7.96(\mathrm{br}$ S., $2 \mathrm{H}, \mathrm{NH}_{2}$ ), 7.78 (d, $2 \mathrm{H}, J=8.0 \mathrm{~Hz}, \mathrm{H}_{\text {arom }}-3^{\prime}+\mathrm{H}_{\text {arom. }}-5^{\prime}$ ), 7.56 (d, $\left.2 \mathrm{H}, J=8.0 \mathrm{~Hz}, \mathrm{H}_{\text {arom }}-2^{\prime}+\mathrm{H}_{\text {arom. }}-6^{\prime}\right), 6.69$ (br s., $\left.2 \mathrm{H}, \mathrm{NH}_{2}\right), 3.71$ (m, $\left.4 \mathrm{H}, 2 \mathrm{xCH}_{2(\text { piperidin) })}\right), 1.65-1.50$ (m, $6 \mathrm{H}, 3 \mathrm{xCH}_{2 \text { (piperidin) }) .}{ }^{13} \mathrm{C} \mathrm{NMR}$ (150.91, DMSO- $\left.d_{6}, \quad \delta, \quad \mathrm{ppm}\right): 162.7 \quad\left(\mathrm{C}(2)_{\text {pyramid. }}\right), \quad 160.8$ (C(4)pyrimid.), 157.0 (C(6)pyrimid.), 132.7, 130.1, 128.6 (Carom.), 123.3 (C-Br), 106.9 (C(5) pyrimid.), 53.0 (2xCH 2 (piperidin)), 25.9, 24.4 (3xCH2(piperidin)). Anal. calcd. for $\mathrm{C}_{15} \mathrm{H}_{18} \mathrm{BrN}_{7}$ : $\mathrm{C}, 47.88 ; \mathrm{H}, 4.82 ; \mathrm{N}$, 26.06. Found: C, $47.59 ; \mathrm{H}, 4.68$; N, $25.89 \%$.

2,6-Diamino-5-((p-bromophenyl)diazenyl)-4-morpholinopyrimidine (12): From morpholine $(87 \mathrm{mg})$. Yield: $166 \mathrm{mg}$ (88\%). M.p.: $238-242{ }^{\circ} \mathrm{C} . R_{\mathrm{f}}=0.58 .{ }^{1} \mathrm{H}$ NMR $\left(400 \mathrm{MHz}\right.$, DMSO- $d_{6}$, $\delta$, ppm): 7.79 (br s., $2 \mathrm{H}, \mathrm{NH}_{2}$ ), $7.62\left(\mathrm{~d}, 2 \mathrm{H}, J=7.9 \mathrm{~Hz}, \mathrm{H}_{\text {arom. }}-3^{\prime}+\right.$ 


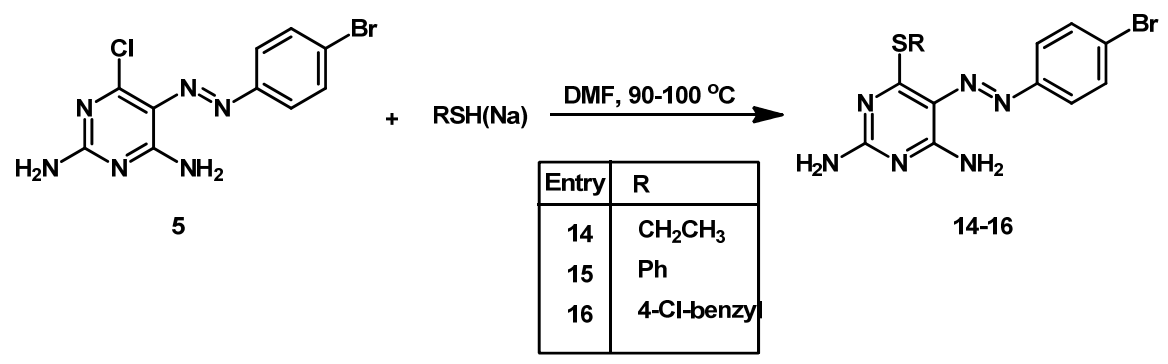

Scheme 2

$\mathrm{H}_{\text {arom. }}-5^{\prime}$ ), 7.53 (d, $2 \mathrm{H}, J=7.9 \mathrm{~Hz}, \mathrm{H}_{\text {arom. }}-2^{\prime}+\mathrm{H}_{\text {arom. }}-6^{\prime}$ ), 6.84 (br s., $\left.2 \mathrm{H}, \mathrm{NH}_{2}\right), 3.88\left(\mathrm{~m}, 4 \mathrm{H} 2 \mathrm{xCH}_{2 \text { (morpholin) }} 3.71\right.$ (m, 4H, $2 \mathrm{xCH}_{2 \text { (morpholin). }}{ }^{13} \mathrm{C}$ NMR (150.91, DMSO- $d_{6}, \delta, \mathrm{ppm}$ ): 163.3 (C(2) pyrimid.), 161.3 (C(4) pyrimid.), 156.8 (C(6)pyrimid.), 132.6, 130.6, 127.5 (Carom.), 122.9 (C-Br), 105.8 (C(5) 10 pyrimid.), 66.9 ( $\left.2 \mathrm{xC}_{\text {morpholin }}\right), 49.6$ ( $2 \mathrm{xC}_{\text {morpholin }}$ ). Anal. calcd. for $\mathrm{C}_{14} \mathrm{H}_{16} \mathrm{BrN}_{7} \mathrm{O}$ : $\mathrm{C}$, 44.46; H, 4.26; N, 25.92. Found: C, 44.19; H, 4.08; N, $25.69 \%$.

2,6-Diamino-5-((p-bromophenyl)diazenyl)-4-piperazinopyrimidine (13): From piperazine (86 mg). Yield: $153 \mathrm{mg}$ (81\%). M.p.: $238-242^{\circ} \mathrm{C} . R_{\mathrm{f}}=0.11 .{ }^{1} \mathrm{H}$ NMR $\left(400 \mathrm{MHz}\right.$, DMSO- $d_{6}$, $\delta$, ppm): 8.07 (br s., $2 \mathrm{H}, \mathrm{NH}_{2}$ ), $7.62\left(\mathrm{~d}, 2 \mathrm{H}, J=7.7 \mathrm{~Hz}, \mathrm{Harom} .3^{\prime}+\right.$ $\mathrm{H}_{\text {arom. }}-5^{\prime}$ ), 7.52 (d, $2 \mathrm{H}, J=7.7 \mathrm{~Hz}, \mathrm{H}_{\text {arom. }}-2^{\prime}+\mathrm{H}_{\text {arom. }}-6^{\prime}$ ), 6.66 (br s., $2 \mathrm{H}, \mathrm{NH}_{2}$ ), 3.30 (br s., $1 \mathrm{H}, \mathrm{NH}$ ), 3.09 (br s., $4 \mathrm{H}, 2 \mathrm{xCH}_{2 \text { (piperazin), }}$

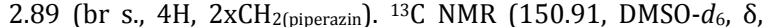

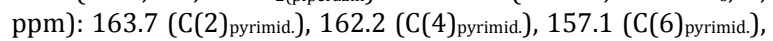
132.7, 130.4, 127.4, (Carom.), 122.9 (C-Br), 105.8 (C(5)pyrimid.),

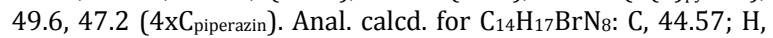
4.54; N, 29.70. Found: C, 44.27; H, 4.40; N, $27.75 \%$.

\subsubsection{General procedure for the preparation of 2,6-diamino 4-alkyl-and arylthio-5-((p-bromophenyl)diazenyl) pyrimidine derivatives (14-16)}

A solution of compound 5 (100 $\mathrm{mg}, 0.31 \mathrm{mmol}$ ) in DMF (20 $\mathrm{mL}$ ) and sodium ethanethiolate, sodium thiophenolate or $p$ chlorobenzenethiol $(0.90 \mathrm{mmol})$ was heated in an oil bath at 90-100 ${ }^{\circ} \mathrm{C}$ for $4-6 \mathrm{~h}$. After cooling, the solution was evaporated and the residue was co-evaporated with toluene $(4 \times 15 \mathrm{~mL})$ to give a crude solid. Recrystallization from ethanol or ethanol:ether $(1: 3, v: v)$ afforded the desired product (Scheme 2).

5-((4-Bromophenyl)diazenyl)-6-(ethylthio)pyrimidine-2,4diamine (14): From sodium ethanethiolate $(76 \mathrm{mg})$. Yield: 81 mg (74\%). M.p.: $236-238{ }^{\circ} \mathrm{C} . R_{\mathrm{f}}=0.63 .{ }^{1} \mathrm{H}$ NMR $(400 \mathrm{MHz}$ DMSO- $d_{6}, \delta, \mathrm{ppm}$ ): 9.26 (br s., $2 \mathrm{H}, \mathrm{NH}_{2}$ ), 8.19 (br s., $2 \mathrm{H}, \mathrm{NH}_{2}$ ), 7.72 (d, $\left.2 \mathrm{H}, J=7.9 \mathrm{~Hz}, \mathrm{H}_{\text {arom. }}-3^{\prime}+\mathrm{H}_{\text {arom. }}-5^{\prime}\right), 7.69$ (d, $2 \mathrm{H}, J=7.9$ Hz, Harom. $-2^{\prime}+\mathrm{H}_{\text {arom. }}-6^{\prime}$ ), 3.99 (q, $\left.2 \mathrm{H}, J=7.1 \mathrm{~Hz}, \mathrm{CH}_{2} \mathrm{CH}_{3}\right), 1.23$ (t, $\left.3 \mathrm{H}, \mathrm{CH}_{2} \mathrm{CH}_{3}\right) .{ }^{13} \mathrm{C}$ NMR (150.91, DMSO- $\left.d_{6}, \delta, \mathrm{ppm}\right): 163.0$

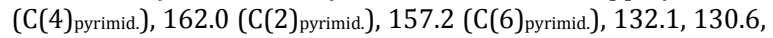
127.9 (Carom.), 121.6 (C-Br), 100.0 (C(5) pyrimid.), $41.6\left(\mathrm{SCH}_{2} \mathrm{CH}_{3}\right.$ ), $11.4\left(\mathrm{SCH}_{2} \mathrm{CH}_{3}\right)$. MS $((+)-\mathrm{FAB}): m / z=352 / 354[\mathrm{M}]^{+}$. Anal. calcd. for $\mathrm{C}_{12} \mathrm{H}_{13} \mathrm{BrN}_{6} \mathrm{~S}: \mathrm{C}, 40.80 ; \mathrm{H}, 3.71 ; \mathrm{N}, 23.79$. Found: $\mathrm{C}, 40.61 ; \mathrm{H}$, $3.60 ; \mathrm{N}, 23.54 \%$.

2,4-Diamino-5-((p-bromophenyl)diazenyl)-6-(phenylthio) pyrimidine (15): From sodium benzenethiolate $(117 \mathrm{mg})$. Yield: $85 \mathrm{mg}(68 \%)$. M.p.: $224-226{ }^{\circ} \mathrm{C}$ (Dec.). $R_{\mathrm{f}}=0.53 .{ }^{1} \mathrm{H}$ NMR $(400$ MHz, DMSO- $d_{6}, \delta$, ppm): 8.32 (br s., $2 \mathrm{H}, \mathrm{NH}_{2}$ ), 8.19 (br s., $2 \mathrm{H}$ $\mathrm{NH}_{2}$ ), 7.90-7.59 (m, 9H, $\mathrm{H}_{\text {arom.). }}{ }^{13} \mathrm{C}$ NMR (150.91, DMSO- $d_{6}, \delta$ ppm): 181.4 (C(4) pyrimid.), 164.6 (C(2) pyrmid.), 156.0 (C(6) pyrimid.) 133.5, 132.2, 132.1, 129.0, 127.8, 123.8 (Carom.), 122.1 (C-Br) 118.1 (C(5)pyrimid.). MS ((+)-FAB): $m / z=401 / 402[\mathrm{M}]^{+}$. Anal. calcd. for $\mathrm{C}_{16} \mathrm{H}_{13} \mathrm{BrN}_{6} \mathrm{~S}$ : C, 47.89; $\mathrm{H}, 3.27$; N, 20.94. Found: C, 47.77; H, 3.16; N, $20.76 \%$.
2,4-Diamino-5-((p-bromophenyl)diazenyl)-6-((4-chloro benzyl)thio)pyrimidine (16): From 4-chlorobenzylthiol (143 mg). Yield: $74 \mathrm{mg}(53 \%)$. M.p.: $232-236{ }^{\circ} \mathrm{C}$ (Dec.). $R_{\mathrm{f}}=0.55 .{ }^{1} \mathrm{H}$ NMR (400 MHz, DMSO- $d_{6}, \delta, \mathrm{ppm}$ ): 9.26 (br s., $2 \mathrm{H}, \mathrm{NH}_{2}$ ), 8.19 (br s., $2 \mathrm{H}, \mathrm{NH}_{2}$ ), 7.75-7.29 (m, 8H, $\mathrm{H}_{\text {arom. }}$ ), $4.36\left(\mathrm{~s}, 2 \mathrm{H}, \mathrm{CH}_{2}\right) .{ }^{13} \mathrm{C}$ NMR (150.91, DMSO- $\left.d_{6}, \delta, \mathrm{ppm}\right): 164.6$ (C(4) $)_{\text {pyrimid.) }}, 161.1$

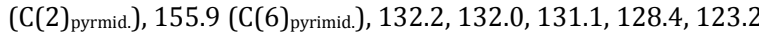
(Carom.), 122.1 (C-Br), 118.6 (C(5) pyrimid.), $31.2\left(\mathrm{CH}_{2}\right)$. MS ((+)FAB): $m / z=448 / 450[\mathrm{M}]^{+}$. Anal. calcd. for $\mathrm{C}_{17} \mathrm{H}_{14} \mathrm{BrClN}_{6} \mathrm{~S}: \mathrm{C}$, 45.40; H, 3.14; N, 18.69. Found: C, 45.21; H, 3.02; N, $18.42 \%$.

\subsubsection{Reaction of compound 17 and 18 with thiourea under MWI}

A crushed mixture of compound 17 (100 mg, $0.46 \mathrm{mmol})$ or $18(100 \mathrm{mg}, 0.71 \mathrm{mmol})$ and thiourea $(10 \mathrm{mg}, 1.31 \mathrm{mmol})$ was irradiated in a microwave oven $(800 \mathrm{~W})$ for $25 \mathrm{~min}$. The mixture was poured into ice-cold water and the solid residue was washed with ether followed by a little amount of cold ethanol. Recrystallization from ethanol afforded the desired product (Scheme 3).

2-Amino-6-(benzyloxy)pyrimidine-4-thione (19): Yield: 76 mg (71\%). M.p.: $136-138{ }^{\circ} \mathrm{C} . R_{\mathrm{f}}=0.57 .{ }^{1} \mathrm{H}$ NMR $(400 \mathrm{MHz}$ DMSO- $d_{6}, \delta, \mathrm{ppm}$ ): 7.42-7.33 (m, $5 \mathrm{H}, \mathrm{H}_{\text {arom. }}$ ), 7.07 (br s., $2 \mathrm{H}_{,}$ $\mathrm{NH}_{2}$ ), 6.15 (s, 1H, NH), 5.31 (s, 1H, Hpyrimid-5), 5.13 (s, 2H, $\mathrm{CH}_{2}$ ). ${ }^{13} \mathrm{C}$ NMR (150.91, DMSO- $\left.d_{6}, \delta, \mathrm{ppm}\right): 184.3$ (C6=S), 170.3

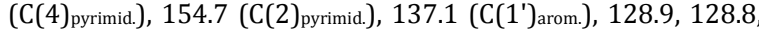
128.4, 128.2 (Carom.), 94.7 (C(5)pyrimid.), $65.7\left(\mathrm{CH}_{2}\right)$. Anal. calcd. for $\mathrm{C}_{11} \mathrm{H}_{11} \mathrm{~N}_{3} \mathrm{OS}$ : C, 56.63; H, 4.75; N, 18.01. Found: $\mathrm{C}, 56.41 ; \mathrm{H}$ $4.65 ; \mathrm{N}, 17.82 \%$.

2-Amino-6-methoxypyrimidine-4-thione (20): Yield: $76 \mathrm{mg}$ (67\%). M.p.: $289-292{ }^{\circ} \mathrm{C}$ (Dec.). $R_{\mathrm{f}}=0.37 .{ }^{1} \mathrm{H}$ NMR $(400 \mathrm{MHz}$ DMSO- $d_{6}, \delta, \mathrm{ppm}$ ): 7.28 (br s., $2 \mathrm{H}, \mathrm{NH}_{2}$ ), $6.76(\mathrm{~s}, 1 \mathrm{H}, \mathrm{NH}$ ), 5.35 (s, $\left.1 \mathrm{H}, \mathrm{H}_{\text {pyrimid }}-5\right), 4.07$ (s, 3H, $\left.\mathrm{OCH}_{3}\right) .{ }^{13} \mathrm{C}$ NMR (150.91, DMSO$d_{6}, \delta, \mathrm{ppm}$ ): 184.9 ( $\left.\mathrm{C}=\mathrm{S}\right), 171.8$ (C(4)pyrimid.), 153.8 (C2) pyrimid.), 94.1 ( $(5)_{\text {pyrimid.), }} 57.0$ (OMe). Anal. calcd. for $\mathrm{C}_{5} \mathrm{H}_{7} \mathrm{~N}_{3} \mathrm{OS}$ : $\mathrm{C}$, 38.20; H, 4.49; N, 26.73. Found: C, 8.43; H, 4.37; N, $26.52 \%$.

\subsubsection{Synthesis of 2-(2-amino-6-methoxypyrimidin-4-yl) mercapto acetic acid (21)}

To a solution of compound 20 ( $55 \mathrm{mg}, 0.34 \mathrm{mmol}$ ) in DMF $(5 \mathrm{~mL})$ containing potassium carbonate $(30 \mathrm{mg})$ was added 2 mercaptoacetic acid $(30 \mathrm{mg}, 0.32 \mathrm{mmol}$ ) and stirred at room temperature for $16 \mathrm{~h}$. After cooling, the mixture was filtered and the filtrated was evaporated to dryness and the residue was purified on a short column of $\mathrm{SiO}_{2}(5 \mathrm{~g})$. Elution with chloroform:methanol $(95: 5, v: v)$ afforded compound 21 (35 mg, $47 \%$ ) (Scheme 3). M.p.: $142-154{ }^{\circ} \mathrm{C}$ (Dec.). $R_{\mathrm{f}}=0.1 .{ }^{1} \mathrm{H}$ NMR (400 MHz, DMSO- $d_{6}, \delta, p p m$ ): 10.95 (s, $1 \mathrm{H}, \mathrm{OH}$ ), 7.23 (br s., $2 \mathrm{H}$ $\left.\mathrm{NH}_{2}\right), 6.33$ (s, $\left.1 \mathrm{H}, \mathrm{H}_{\text {pyrimid. }}-5\right), 4.08\left(\mathrm{~s}, 2 \mathrm{H}, \mathrm{CH}_{2}\right), 3.88(\mathrm{~s}, 3 \mathrm{H}$ OMe). ${ }^{13} \mathrm{C}$ NMR (150.91, DMSO- $\left.d_{6}, \delta, \mathrm{ppm}\right): 71.7\left(\mathrm{CO}_{2} \mathrm{H}\right), 170.6$

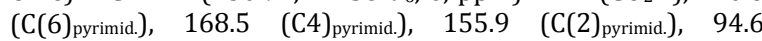
(C(5)pyrimid.), $51.9(\mathrm{OMe}), 31.0\left(\mathrm{CH}_{2}\right)$. 


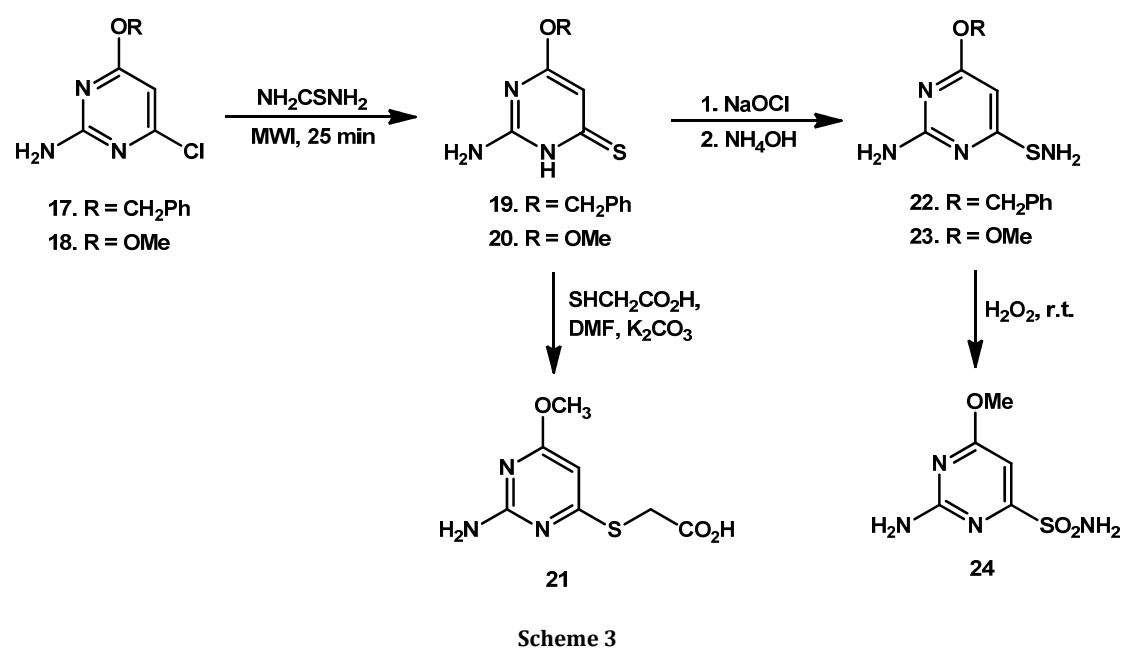

Anal. calcd. for $\mathrm{C}_{7} \mathrm{H}_{9} \mathrm{~N}_{3} \mathrm{O}_{3} \mathrm{~S}$ : C, 39.06; $\mathrm{H}, 4.21 ; \mathrm{N}, 19.52$. Found: C, 38.82; $\mathrm{H}, 4.10$; N, 19.32 .

\subsubsection{Reaction of compound 21 with sodium hypochlorite and ammonium hydroxide}

Compound 21 (104 mg, $0.66 \mathrm{mmol}$ ) or 20 (54 mg, 0.34 mmol) was added to a solution of sodium hypochlorite (5 mL) and the reaction mixture was stirred at $60{ }^{\circ} \mathrm{C}$ for $4 \mathrm{~h}$. Ammonium hydroxide $(10 \mathrm{~mL})$ was added to the reaction mixture, and stirred for an additional $1 \mathrm{~h}$ at room temperature, the precipitate was filtered and washed with water several times and dried, followed by washing with dry ether to give compound $\mathbf{2 2}$ or $\mathbf{2 3}$, respectively (Scheme 3 ).

2-Amino-4-(aminothio)-6-(benzyloxy)pyrimidine (22): Yield: $111 \mathrm{mg}(68 \%)$. M.p.: $286-289^{\circ} \mathrm{C} . R_{\mathrm{f}}=0.32 .{ }^{1} \mathrm{H}$ NMR $(400 \mathrm{MHz}$, DMSO- $d_{6}, \delta, \mathrm{ppm}$ ): 7.43-7.33 (m, $5 \mathrm{H}, \mathrm{H}_{\text {arom.) }}$ ) 7.33 (br s., $2 \mathrm{H}$, $\mathrm{NH}_{2}$ ), 6.14 (s, 1H, Hpyrimid.-5), 5.39 (br s., $2 \mathrm{H}, \mathrm{SNH}_{2}$ ), 5.31 (s, 2H, $\left.\mathrm{CH}_{2}\right) .{ }^{13} \mathrm{C}$ NMR (150.91, DMSO- $\left.d_{6}, \delta, \mathrm{ppm}\right): 189.4\left(\mathrm{CSNH}_{2}\right), 166.1$ (C(4) pyrimid.), 160.5 (C2) pyrimid.), 129.0-126.9 (Carom.), 100 (C(5)pyrimid.). Anal. calcd. for $\mathrm{C}_{11} \mathrm{H}_{12} \mathrm{~N}_{4} \mathrm{OS}$ : C, 53.21; $\mathrm{H}, 4.87 ; \mathrm{N}$, 22.56. Found: C, 52.98; H, 4.70; N, $22.68 \%$.

2-Amino-4-(aminothio)-6-methoxypyrimidine (23): Yield: 36 mg (61\%). M.p.: $288-291{ }^{\circ} \mathrm{C} . R_{\mathrm{f}}=0.43 .{ }^{1} \mathrm{H}$ NMR $(400 \mathrm{MHz}$, DMSO- $d_{6}, \delta, \mathrm{ppm}$ ): 7.41 (br s., $2 \mathrm{H}, \mathrm{NH}_{2}$ ), 5.99 (s, $1 \mathrm{H}, \mathrm{H}_{\text {pyrimid. }}-5$ ), 3.87 (s, 3H, OMe), 2.31 (s, 2H, $\mathrm{SNH}_{2}$ ). ${ }^{13} \mathrm{C}$ NMR (150.91, DMSO-

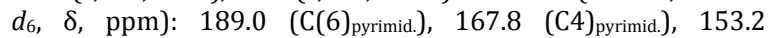
(C(2) pyrimid.), 94.9 (C( 5 pyrimid.), 57.0 (OMe). Anal. calcd. for $\mathrm{C}_{5} \mathrm{H}_{8} \mathrm{~N}_{4} \mathrm{OS}$ : C, 34.87; $\mathrm{H}, 4.68 ; \mathrm{N}, 32.53$. Found: $\mathrm{C}, 34.59 ; \mathrm{H}, 4.55$; $\mathrm{N}, 32.33 \%$.

\subsubsection{Synthesis of 2-amino-6-methoxypyrimidine-4- sulfonamide (24)}

A suspension of compound 23 (80 mg, $0.46 \mathrm{mmol}$ ) in $\mathrm{H}_{2} \mathrm{O}_{2}$ $(5 \mathrm{~mL})$ was stirred at room temperature for $4 \mathrm{~h}$. The solution was evaporated to dryness and the residue was washed with water $(3 \times 10 \mathrm{~mL})$, dried and then then with ether. The dried crude product was purified on a short $\mathrm{SiO}_{2}$ column $(5 \mathrm{~g})$ using chloroform:methanol $(4: 1, v: v)$ as eluent, to give compound 24 (70 mg, 74\%) (Scheme 3). M.p.: 156-158 ${ }^{\circ} \mathrm{C} .1^{1} \mathrm{H}$ NMR (400 MHz, DMSO- $d_{6}, \delta, \mathrm{ppm}$ ): 7.32 (br s., $2 \mathrm{H}, \mathrm{NH}_{2}$ ), 6.51 (s, $1 \mathrm{H}, \mathrm{H}_{\text {pyrimid. }}-5$ ), 3.98 (s, $3 \mathrm{H}, \mathrm{OMe}$ ), 2.11 (br s., $2 \mathrm{H}, \mathrm{SO}_{2} \mathrm{NH}_{2}$ ). ${ }^{13} \mathrm{C}$ NMR (150.91, DMSO- $\left.d_{6}, \delta, \mathrm{ppm}\right): 168.9$ (C(6) $\left.)_{\text {pyrimid. }}\right), 165.1$ (C4) $)_{\text {pyrimid.), }} 154.7$ (C(2) pyrimid.), 96.2 (C(5) pyrimid.), 57.2 (OMe). Anal. calcd. for $\mathrm{C}_{5} \mathrm{H}_{8} \mathrm{~N}_{4} \mathrm{O}_{3} \mathrm{~S}$ : C, 29.41; H, 3.95; N, 27.44. Found: C, 29.19; H, 3.86; $\mathrm{N}, 27.21 \%$.

\section{Results and discussion}

\subsection{Chemistry}

Recently, reported the synthesis of azopyrimidine 5 [33], and selected here as a starting material for the synthesis of new azopyrimidine analogues having alkyl and aryl amino groups at C-4. Thus, compound $\mathbf{5}$ was subjected to a nucleophilic displacement of chlorine group by treatment with various amines in DMF at $90-100{ }^{\circ} \mathrm{C}$ for $4-5 \mathrm{~h}$, leading to the new derivatives 6-13 in good to moderate yields (55-89\%), except the products $\mathbf{7}$ and $\mathbf{1 0}$ were obtained in a lower yields 32 and $36 \%$, respectively (Scheme 1), might due to the steric factor (Scheme 1).

The structures of 6-13 were established by ${ }^{1} \mathrm{H},{ }^{13} \mathrm{C}$ NMR and mass spectral data. In the ${ }^{1} \mathrm{H}$ NMR spectra, both amino groups at C-2 and C- 6 of pyrimidine backbone appeared almost at the same regions as broad singlets at $\delta 6.84-6.38 \mathrm{ppm}$ and $\delta 10.61$ $7.79 \mathrm{ppm}$, respectively, assigned by $\mathrm{D}_{2} \mathrm{O}$ exchange. The amino groups at C- 6 of the analogue 6 showed two broad singlets $(\delta$ 8.85 and $8.14 \mathrm{ppm}$ ) and not doublets as expected, might be due to the hydrogen bonding with the nitrogen of the azo group at C-5. The aromatic and the aliphatic protons were fully analyzed (c.f. Experimental section). In the ${ }^{13} \mathrm{C}$ NMR spectra of 6-13, C-2 and $\mathrm{C}-4$ of the pyrimidine scaffold resonated at the region $\delta$ $164.0-163.2 \mathrm{ppm}$ and the region $\delta$ 162.3-160.0 ppm, respectively. C-5 and C- 6 appeared at the regions $\delta 110.1-100.0$ and 157.7-152.0 ppm, respectively. The aromatic carbon atoms C-4' carrying bromine residue were resonated the regions $\delta$ 123.8-118.9 ppm, while the aromatic carbon atoms C-1' - C-6' of the pyrimidine analogues 6-13 were resonated at the regions $\delta$ 140.8-122.6 ppm. The aliphatic carbon atoms were fully analyzed ( $c f$ Experimental section).

Next, the analogue $\mathbf{5}$ was used as a precursor for the synthesis of new 5-arylazo-4-ethyl(aryl)thio-pyrimidine derivatives to examine their antiviral activity in comparison to the azoaryl analogues 6-13. Thus, treatment of compound 5 with sodium ethanethiolate, sodium thiophenolate, or $p$-chlorobenzylthiol in DMF afforded, via nucleophilic displacements of the chlorine group, 14-16 in 74, 68 and 53\% yield, respectively (Scheme 2).

The structures of compounds 14-16 were assigned by the ${ }^{1} \mathrm{H}$ and ${ }^{13} \mathrm{C}$ NMR spectra. The ${ }^{1} \mathrm{H}$ NMR spectra showed rather similar patterns for the phenyl and ethyl protons. The methylene and methyl protons of SEt group appeared as quartet and triplet at $\delta 3.99$ and $1.36 \mathrm{ppm}(J=7.1 \mathrm{~Hz}$ ), respectively, whereas the aromatic protons were fully analysed ( $c f$ Experimental section). 


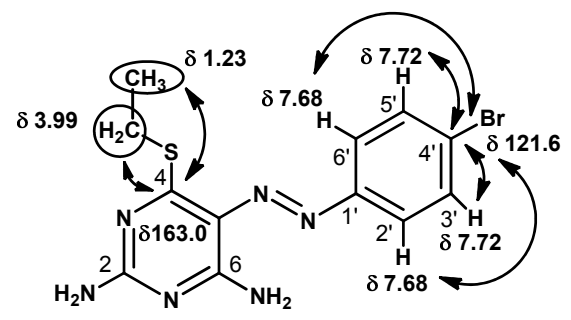

Figure 2. $J_{C, H}$ Correlations in the HMBC NMR spectrum of compound 14.

In the ${ }^{13} \mathrm{C}$ NMR spectra of compounds 14-16, C-4, C-5 and C-6 together with the aromatic carbon atoms were identified. However, compound 14 has been selected for further NMR experiment. In the gradient-selected HMBC spectrum [34] of compound 14, C-4 of the pyrimidine ring at $\delta 163.0 \mathrm{ppm}$ showed a ${ }^{2} \mathrm{~J}$, $\mathrm{H}$ coupling with methylene protons of $\mathrm{CH}_{3} \mathrm{CH}_{2} \mathrm{~S}$ group at $\delta 3.99 \mathrm{ppm}$, in addition to a ${ }^{3} \mathrm{C}_{\mathrm{C}, \mathrm{H}}$ coupling with the methyl protons of the $\mathrm{CH}_{3} \mathrm{CH}_{2} \mathrm{~S}$ group at $\delta 1.23$ ppm. Further, the aromatic protons $\mathrm{H}-3^{\prime}$ and $\mathrm{H}-5^{\prime}$ at $\delta 7.72 \mathrm{ppm}$ revealed two ${ }^{2} J \mathrm{C}, \mathrm{H}$ coupling with $\mathrm{C}-4{ }^{\prime}(\mathrm{C}-\mathrm{Br})$ of the aromatic ring at $\delta 121.6$ ppm, while the same carbon atom $(\mathrm{C}-\mathrm{Br})$ showed two ${ }^{3} \mathrm{~J}_{\mathrm{C}, \mathrm{H}}$ couplings with $\mathrm{H}-2^{\prime}$ and $\mathrm{H}-6^{\prime}$ of the same ring at $\delta 7.68 \mathrm{ppm}$ (Figure 2).

Our work was modified by conversion of the chloro residue in the pyrimidine scaffold to the thione, following Lawson and Tankler method [35]. Thus, compound $\mathbf{1 7}$ and $\mathbf{1 8}$ were treated with thiourea under microwave irradiation (MWI) in a free solvent condition for 15 min afforded, after purification, the pyrimidine-thione analogues 19 and 20 in 71 and $67 \%$ yield, respectively. Treatment of compound 20 with 2-mercapto acetic acid in DMF in the presence of $\mathrm{K}_{2} \mathrm{CO}_{3}$ at room temperature for $16 \mathrm{~h}$ afforded regioselectively [36], after chromatographic purification, 4-thio-ethylacetic acid derivative $21(47 \%)$.

Next, treatment of compound 19 and 20 with sodium hypochlorite followed by ammonium hydroxide, following Rice et al. method [37], furnished after purification, the 4aminothio-pyrimidine derivatives 22 and 23 in 61 and 68\% yield, respectively. Oxidation of compound 23 with $\mathrm{H}_{2} \mathrm{O}_{2}$ at room temperature afforded, after chromatographic purifycation, the sulfonamide $\mathbf{2 4}$ in $\mathbf{7 4 \%}$ yield (Scheme 3).

The structures of compound 19-24 were elucidated from their ${ }^{1} \mathrm{H}$ and ${ }^{13} \mathrm{C}$ NMR spectra. In the ${ }^{1} \mathrm{H}$ NMR spectra of compounds 19 and 20, $\mathrm{H}-5$ of the pyrimidine ring were appeared at $\delta 5.31$ and $5.39 \mathrm{ppm}$, respectively, whereas the protons of the amino groups at $\mathrm{C}-2$ were resonated as broad singlet at $\delta 7.07$ and $7.33 \mathrm{ppm}$, respectively. The $\mathrm{SNH}_{2}$ protons of compound 22 resonated as a broad singlet at $\delta 5.39 \mathrm{ppm}$, while the singlet and broad singlet at $\delta 6.76$ and $2.31 \mathrm{ppm}$ were assigned to $\mathrm{NH}$ proton of the pyrimidine thione $\mathbf{2 0}$ and the $\mathrm{SNH}_{2}$ protons at C-4 of the analogue 23, respectively. The singlets at $\delta 5.35$ and $5.99 \mathrm{ppm}$ were attributed to $\mathrm{H}-5$ of the pyrimidine ring, in addition to two singlets at $\delta 4.07$ and 4.09 ppm, assigned to the methoxy group at C-6 of compound 20 and 23, respectively. Compound 24 showed singlet and broad singlet at $\delta 6.51$ and $2.11 \mathrm{ppm}$, identified as $\mathrm{H}-5$ and $\mathrm{SO}_{2} \mathrm{NH}_{2}$ protons, respectively. In the ${ }^{13} \mathrm{C}$ NMR of compound 19-24, the resonances at the lower field regions $\delta$ 189.4-168.9 ppm were assigned to C-6 (C-S) of the pyrimidine ring in comparison for those of the starting materials $\mathbf{1 7}$ and $\mathbf{1 8}$ at $\delta 164.1$ and 162.7 ppm, respectively. C-2, C-4 and C-5 together with the substituents carbon atoms were fully identified ( $c f$ Experimental section). All the synthesized were confirmed also from their ${ }^{1} \mathrm{H},{ }^{13} \mathrm{C}$ HSQC [38] NMR spectra.

\subsection{In vitro anti-HIV activity}

Compounds 6-13, 14-16 and 19-24 were tested for their in vitro anti-HIV-1 (strain $\mathrm{III}_{\mathrm{B}}$ ) and HIV-2 (strain ROD) activity in human T-lymphocyte (MT-4) cells based on an MTT assay [39]. The results are summarized in Table 1, in which the data for nevirapine (BOE/BIRG587) [40] and azidothymidine (DDN/AZT) [41] were included for comparison.

Table 1. In-vitro anti-HIV-1a and HIV-2 b of new pyrimidine analogues 4-16 and 19-27.

\begin{tabular}{|c|c|c|c|c|}
\hline Compound & Virus strain & $\begin{array}{l}\mathrm{EC}_{50} \\
(\mu \mathrm{g} / \mathrm{mL})^{\mathrm{c}}\end{array}$ & $\begin{array}{l}\mathrm{CC}_{50} \\
(\mu \mathrm{g} / \mathrm{mL})^{\mathrm{d}}\end{array}$ & SI e \\
\hline \multirow[t]{2}{*}{$\overline{6}$} & $\mathrm{III}_{\mathrm{B}}$ & $>45.23$ & 45.23 & $<1$ \\
\hline & ROD & $>45.23$ & 45.23 & $<1$ \\
\hline \multirow[t]{2}{*}{7} & $\mathrm{III}_{\mathrm{B}}$ & $>65.23$ & 65.23 & $<1$ \\
\hline & ROD & $>65.23$ & 65.23 & $<1$ \\
\hline \multirow[t]{2}{*}{8} & $\mathrm{III}_{\mathrm{B}}$ & $>32.60$ & 32.60 & $<1$ \\
\hline & ROD & $>32.60$ & 32.60 & $<1$ \\
\hline \multirow[t]{2}{*}{9} & $\mathrm{III}_{\mathrm{B}}$ & $>53.60$ & 53.60 & $<1$ \\
\hline & ROD & $>53.60$ & 53.60 & $<1$ \\
\hline \multirow[t]{2}{*}{10} & $\mathrm{III}_{\mathrm{B}}$ & $>35.10$ & 35.10 & $<1$ \\
\hline & ROD & $>35.10$ & 35.10 & $<1$ \\
\hline \multirow[t]{2}{*}{11} & $\mathrm{III}_{\mathrm{B}}$ & $>12.35$ & 12.35 & $<1$ \\
\hline & ROD & $>12.35$ & 12.35 & $<1$ \\
\hline \multirow[t]{2}{*}{12} & $\mathrm{III}_{\mathrm{B}}$ & $>28.05$ & 28.05 & $<1$ \\
\hline & ROD & $>28.05$ & 28.05 & $<1$ \\
\hline \multirow[t]{2}{*}{13} & $\mathrm{III}_{\mathrm{B}}$ & $>10.04$ & 10.04 & $<1$ \\
\hline & ROD & $>10.04$ & 10.04 & $<1$ \\
\hline \multirow[t]{2}{*}{14} & $\mathrm{III}_{\mathrm{B}}$ & $>2.12$ & 2.12 & $<1$ \\
\hline & ROD & $>2.12$ & 2.12 & $<1$ \\
\hline \multirow[t]{2}{*}{15} & $\mathrm{III}_{\mathrm{B}}$ & $>1.99$ & 1.99 & $<1$ \\
\hline & ROD & $>1.99$ & 1.99 & $<1$ \\
\hline \multirow[t]{2}{*}{16} & $\mathrm{III}_{\mathrm{B}}$ & $>1.80$ & 1.80 & $<1$ \\
\hline & ROD & $>1.80$ & 1.80 & $<1$ \\
\hline \multirow[t]{2}{*}{19} & $\mathrm{III}_{\mathrm{B}}$ & $>11.34$ & 11.34 & $<1$ \\
\hline & ROD & $>11.34$ & 11.34 & $<1$ \\
\hline \multirow[t]{2}{*}{20} & $\mathrm{III}_{\mathrm{B}}$ & $>5.67$ & 5.67 & $<1$ \\
\hline & ROD & $>5.67$ & 5.67 & $<1$ \\
\hline \multirow[t]{2}{*}{21} & $\mathrm{III}_{\mathrm{B}}$ & $>1.92$ & 1.92 & $<1$ \\
\hline & ROD & $>1.92$ & 1.92 & $<1$ \\
\hline \multirow[t]{2}{*}{22} & III $_{\mathrm{B}}$ & $>3.38$ & 3.38 & $<1$ \\
\hline & ROD & $>3.38$ & 3.38 & $<1$ \\
\hline \multirow[t]{2}{*}{23} & $\mathrm{III}_{\mathrm{B}}$ & $>5.98$ & 5.98 & $<1$ \\
\hline & ROD & $>5.98$ & 5.98 & $<1$ \\
\hline \multirow[t]{2}{*}{24} & $\mathrm{III}_{\mathrm{B}}$ & $>10.18$ & 10.18 & $<1$ \\
\hline & ROD & $>10.18$ & 10.18 & $<1$ \\
\hline$\overline{\mathrm{AZT}}$ & $\begin{array}{l}\mathrm{III}_{\mathrm{B}} \\
\mathrm{ROD}\end{array}$ & $\begin{array}{l}0.0022 \\
>25\end{array}$ & $\begin{array}{l}>25 \\
>25\end{array}$ & $\begin{array}{l}>11363 \\
>26596\end{array}$ \\
\hline Nevirapine & $\begin{array}{l}\mathrm{III}_{\mathrm{B}} \\
\mathrm{ROD} \\
\end{array}$ & $\begin{array}{l}0.050 \\
>4.00 \\
\end{array}$ & $\begin{array}{l}0.050 \\
>4.00 \\
\end{array}$ & $\begin{array}{l}>80 \\
<1\end{array}$ \\
\hline
\end{tabular}

a Anti-HIV-1 activity measured with strain $\mathrm{III}_{\mathrm{B}}$

b Anti-HIV-2 activity measured with strain ROD.

c Compound concentration required to achieve $50 \%$ protection of MT-4 cells from the HIV-1 and 2-induced cytopathogenic effect.

d Compound concentration that reduces the viability of mock-infected MT-4 cells by $50 \%$.

e SI: selectivity index $\left(\mathrm{CC}_{50} / \mathrm{EC}_{50}\right)$.

Compounds-induced cytotoxicity was also measured in MT4 cells parallel with the antiviral activity. All compounds are inactive except compounds 14-16 and 21 which showed EC50 values of $>2.12,1.99,1.80$ and $1.92 \mu \mathrm{g} / \mathrm{mL}$, respectively, but no selectivity was observed $(\mathrm{SI}<1)$. 

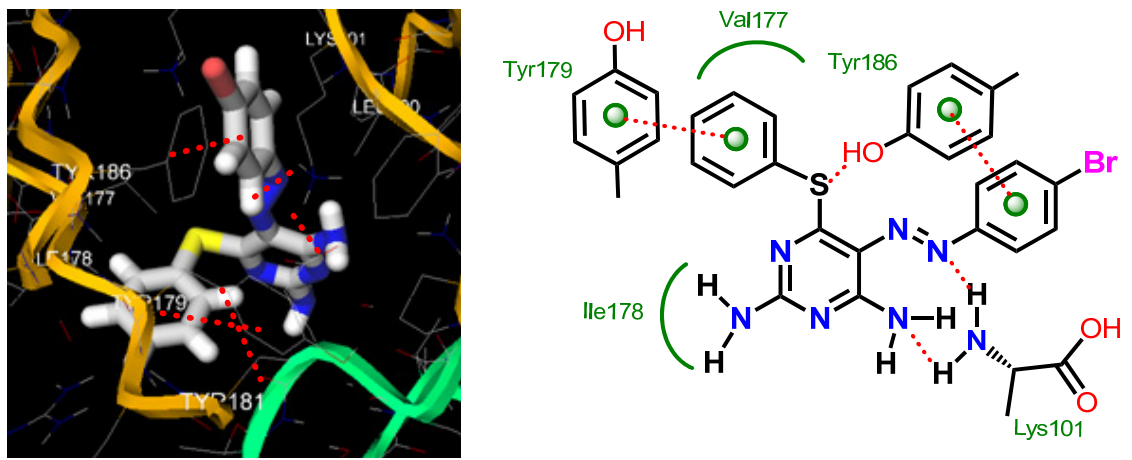

Figure 3. Docked conformation of compound $\mathbf{1 5}$ showing three hydrogen bonding: Lys 101 with $\mathrm{NH}_{2}$ at $\mathrm{C}-6$ and $\mathrm{N}=\mathrm{N}$ groups of the pyrimidine ring, in addition to Tyr186 with sulfur atom of the phenylthio group. It exhibited also hydrophobic interactions between phenyl moieties and Tyr179, Tyr186 of reverse transcriptase (RT) enzyme residues.

From the SAR analysis, we found that the alkyl- or arylthio substituents at $\mathrm{C}-4$ of the pyrimidine ring, e.g. compounds 1416 or thioalkyl acetic acid moiety at C-6 of the same ring, e.g.: compound $\mathbf{2 1}$ were well tolerated in the hydrophobic region of HIV RT and then showed higher activity than those of the alkylor arylamino substituents at $\mathrm{C}-4$ of the same ring; $e$. $g$.: compounds 6-13, and resulted in loss of activity. This means that the thio groups targeting the hydrophobic binding pocket of HIV-1 RT.

\subsection{Molecular modeling analysis}

The molecular docking was performed using SYBYL-X 1.1 and the docking results were shown by PyMOL [42]. Our molecular docking analysis of the new analogues based on the modeling study, which was performed to understand the binding mode of these analogues with the HIV-RT binding pocket (NNIBP) (PDB code: 3DLG, [43]).

Compound 15 has been selected for the docking modeling study, since its binding energy score -8.01, indicating a selectivity and potency profiles of substituted aryl-azopyrimidine to bind the active site of HIV-RT pocket (Figure 3). As shown in figure 3, the aromatic rings of compound 15 fitted into an aromatic rich subpocket surrounded by the aromatic side chains of Tyr179 and Tyr186. The pyrimidine backbone was located in the middle of the binding pocket, anchoring the phenylthio substituent at $\mathrm{C}-4$ in a favourable position for hydrogen bonding with the $\mathrm{OH}$ group of Tyr186 and other two hydrogen bonding of amino group at $\mathrm{C}-6$ and azo group $(\mathrm{N}=\mathrm{N})$ at C-5 with Lys103 of the RT enzyme. Overall, the combination of hydrophobic interaction and $\pi$-stacking appears to govern the binding of compound $\mathbf{1 5}$ with HIV RT.

\section{Conclusion}

In conclusion, synthesis of new 2,6-diamino-4-alkylamino5-p-bromophenylazopyrimidine derivatives, 6-13, the corresponding 4-ethyl- and arylthio analogues, 14-16, and the 4-thio derivatives 19-25 has been described. All the new synthesized compounds have been evaluated for their activity against HIV-1 and 2. Compounds 14-16 and 21 exhibited potential activity against HIV-1, whereas the others analogues shown moderate to poor activity. Compound $\mathbf{1 5}$ have been selected for the molecular modeling study showing its binding to the reverse transcriptase enzyme pocket through three hydrogen bonding and two hydrophobic interactions.

\section{Acknowledgement}

We thank Prof. Christophe Pannecouque of Rega Institute for Medical Research, Katholieke Universiteit, Leuven, Belgium for the anti-HIV screening. Mr. Ulrich Haunz and Miss Anka
Friemel of chemistry department, University of Konstanz, Germany are highly acknowledged for the NMR experiments.

\section{References}

[1]. Cocco, M. T.; Congiu, C.; Lilliu, V. Bioorg. Med. Chem. 2006, 14, 366372.

[2]. Heidelberger, C.; Chaudhuri, N. K.; Danneberg, P.; Mooren, D.; Griesbach, L.; Duschinsky, R.; Schnitzer, R. J.; Pleven, E.; Scheiner, J Nature 1957, 179, 663-666.

[3]. Beattie, J. F.; Breault, G. A.; Ellston, R. P. A.; Green, S.; Jewsbury, P. J.; Midgley, C. J.; Naven, R. T.; Minshull, C. A.; Pauptit, R. A.; Tucker, J. A.; Pease, J. E. Bioorg. Med. Chem. Lett. 2003, 13, 2955-2960.

[4]. Kimura, H.; Katoh, T.; Kajimoto, T.; Node, M.; Hisaki, M.; Sugimoto, Y.; Majima, T.; Uehara, Y.; Yamori, T. Anticancer Res. 2006, 26, 91-97.

[5]. Lagoja, I. M. Chem. Biodiver. 2005, 2, 1-50.

[6]. Dudhea, R.; Sharmab, P. K.; Vermae, P.; Chaudhary, A. J. Adv. Sci. Res. 2011, 2, 10-17.

[7]. Patel, D. H.; Mistry, B. D.; Desai, K. R Indian J. Hetero. Chem. 2003, 13, 179-80

[8]. Bantawal, S. H.; Manjathuru, M.; Mari, K. S.; Padiyath, K. M. Bioorg Med. Chem. 2006, 14, 2040-2047.

[9]. Sharma, P.; Rane, N.; Gurram, V. K. Bioorg. Med. Chem. Lett. 2004, 14 4185-4190.

[10]. Adnan, A. B.; Hesham, T. Z.; Sherif, A. F.; Azza, M. B. Eur. J. Med. Chem. 2003, 38, 27-36.

[11]. Russell, R. K.; Press, J. B.; Rampulla, R. A.; McNally, J. J.; Falotico, R. Keiser, J. A.; J. Med. Chem. 1988, 31, 1786-1793.

[12]. Fillios, L. C.; Naito, C.; Andrews, S.; Roach, A. M. Circ. Res. 1960, 8, 7177.

[13]. Kappe, C. O. Tetrahedron 1993, 49, 6937-6963.

[14]. Kreutzberger, A.; Burgwitz, K. Arch. Pharm. 1981, 314, 394-398.

[15]. Monge, A.; Martinez-Merino, V.; Sanmartin, C.; Fernandez, F. J.; Ochoa M. C.; Bellver, C. Artigas, P. Arznei-Forschung. 1990, 40, 1230-1233.

[16]. Yamazi, Y.; Takahashi, M.; Todome, Y. Proc. Soc. Exp. Biol. Med. 1970 133, 674-677.

[17]. Prichard, M. N.; Quenelle, D. C.; Hartline, C. B.; Harden, E. A.; Jefferson, G.; Frederick, S. L.; Daily, S. L.; Whitley, R. J.; Tiwari, K. N.; Maddry, J. A.; Secrist, J. A.; Kern, E. R. Antimicrob. Agents Chemother. 2009, 53, 52515285.

[18]. Miyasaka, T.; Tanaka, H.; Baba, M.; Hayakawa, H.; Walker, R. T.; Balzarini, J.; De Clercq, E. J. Med. Chem. 1989, 32, 2507-2509.

[19]. Tanaka, H.; Takashima, H.; Ubasawa, M.; Sekiya, K.; Nitta, I.; Baba, M. Shigeta, S.; Walker, R. T.; De Clercq, E.; Miyasaka, T. J. Med. Chem. 1992, 35, 337-345.

[20]. Balzarini, J.; Baba, M.; De Clercq, E. Antimicrob. Agents Chemother 1995, 39, 998-1002.

[21]. Janssen, P. A. J.; Lewi, P. J.; Arnold, E.; Daeyaert, F.; de Jonge, M. Heeres, J.; Koymans, L.; Vinkers, M.; Guillemont, J.; Pasquier, E.; Kukla, M.; Ludovici, D.; Andries, K.; de Béthune, M. -P.; Pauwels, R.; Das, K.; Clark Jr. , A. D.; Frenkel, Y. V.; Hughes, S. H.; Medaer, B.; De Knaep, F.; Bohets, H.; De Clerck, F.; Lampo, A.; Williams, P.; Stoffels, P. J. Med. Chem. 2005, 48, 1901-1909.

[22]. Ludovici, D. W.; de Corte, B. L.; Kukla, M. J.; Ye, H.; Ho, C. Y.; Lichtenstein, M. A.; Kavash, R. W.; Andries, K.; de Bethune, M. P.; Azijn, H.; Pauwels, R.; Lewi, P. J.; Heeres, J.; Koymans, L. M.; de Jonge, M. R. Aken, K. J. V.; Daeyaert, F. F.; Das, K.; Arnold, E.; Janssen, P. A. Bioorg Med. Chem. 2001, 11, 2235-2239.

[23]. Das, K.; Clark, A. D.; Lewi, P. J.; Heeres, J.; de Jonge, M. R.; Koymans, L. M. H.; Vinkers, H. M.; Daeyaert, F.; Ludovici, D. W.; Kukla, M. J.; De Corte, B.; Kavash, R. W.; Ho, C. Y.; Ye, H.; Lichtenstein, M. A.; Andries, 
K.; Pauwels, R.; de Béthune, M. -P.; Boyer, P. L.; Clark, P.; Hughes, S. H.; Janssen, P. A. J.; Arnold, E. J. Med. Chem. 2004, 47, 2550-2560.

[24]. Yan, Z. H.; Wu, H. Q.; Chen, W. X.; Piao, H. R.; He, Q. Q.; Chen, F. E.; De Clercq, E.; Pannecouque, C. Bioorg. Med. Chem. 2014, 22, 3220-3226.

[25]. Pindola, V. K.; Zarowitz, B. J. Pharmacother. 2002, 22, 1249-1265.

[26]. Gong, B.; Hong, F.; Kohm, K.; Jenkins, S.; Tulinsky, J.; Bhatt, R.; de Vries, P.; Singer, J. W.; Klein, P. P. Bioorg. Med. Chem. Lett. 2004, 14, 23032308.

[27]. Mayer, T. U.; Kapoor, T. M.; Haggarty, S. J.; King, R. W.; Schreiber, S. L.; Mitchison, T. J. Science 1999, 286, 971-974.

[28]. Sharp, D. J.; Rogers, G. C.; Scholey, J. M. Nature 2000, 407, 41-47.

[29]. Yoon, Y. A.; Park, C. S.; Cha, M. H.; Choi, H.; Sim, J. Y.; Kim, J. G. Biooorg. Med. Chem. Lett. 2010, 20, 5735-5738.

[30]. Jain, K. S.; Chitre, T. S.; Miniyar, P. B.; Kathiravan, M. K.; Bendre, V. S.; Veer, V. S.; Shahane, S. R.; Shishoo, C. J. Curr. Sci. 2006, 90, 793-803.

[31]. Al-Masoudi, N. A.; Jafar, N. N. A.; Baqir, S. J.; Pannecouque, C.; Leyssen, P.; Neyts, J. Antivir. Chem. Chemother. 2012, 23, 103-112.

[32]. Al-Masoudi, N. A.; Kassim, A. G.; Abdul-Reda, N. A. Nucleos. Nucleot. Nucl. 2014, 33, 141-161.

[33]. Al-Masoudi, N. A.; Marich, Y. A.; Al-Salihi, N. J.; Saeed, B. Z. Naturforsch. 2014, 69b, 913-923.

[34]. Davis, A. L.; Keeler, J.; Laue, E. D.; Moskau, D. J. Magn. Reson. 1992, 98, 207-216.

[35]. Lawson, A.; Tinkler, R. B. Chem. Rev. 1970, 70, 593-618.

[36]. Xu, Y.; Yang, H.; Hu, J.; Wang, X. W; Liu, J. Y. J. Chinese Pharm. Sci. 2007, 16, 125-127.

[37]. Iijima, I.; Rice, K. C. J. Heterocycl. Chem. 1978, 15, 1527-1528.

[38]. Davis, A. L.; Keeler, J.; Laue, E. D.; Moskau, D. J. Magn. Reson. 1992, 98 , 207-216.

[39]. Pauwels, R.; Balzarini, J.; Baba, M.; Snoeck, R.; Schols, D.; Herdewijn, P.; Desmyter, J.; De Clercq, E. J. Virol. Methods 1988, 20, 309-321.

[40]. Hargrave, K. D.; Proudfoot, J. R.; Grozinger, K. G.; Cullen, E.; Kapadia, S. R.; Patel, U. R.; Fuchs, V. U.; Mauldin, S. C.; Vitous, J.; Behnke, M. L.; Klunder, J. M.; Pal, K.; Skiles, J. W.; McNeil, D. W.; Rose, J. M.; Chow, G. C.; Skoog, M. T.; Wu, J. C.; Schmidt, G.; Engel, W. W.; Eberlein, W. G.; Saboe, T. D.; Campbell, S. J.; Rosenthal, A. S.; Adams, J. J. Med. Chem. 1991, 34, 2231-2241.

[41]. Mitsuya, H.; Weinhold, K. J.; Furman, P. A.; Clair, M. H. St.; Lehrmann, S. N.; Gallo, R.; Bolognesi, D.; Barry, D. W.; Broder, S. Proc. Natl. Acad. Sci. USA 1985, 82, 7096-7100.

[42]. Seeliger, S.; de Groot, B. L. J. Comput Aid. Mol. Des. 2010, 24, 417-422

[43]. Zhan, P.; Liu, X.; Li, Z.; Fang, Z.; Pannecouque, C.; De Clercq, E. Chem. Biodivers. 2010, 7, 1717-1727. 